\title{
Multiple antigenic polypeptide composed of heparanase B-cell epitopes shrinks human hepatocellular carcinoma in mice
}

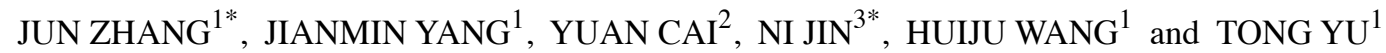 \\ ${ }^{1}$ Department of Gastroenterology, Zhejiang Provincial People's Hospital, Hangzhou, Zhejiang 310014; \\ ${ }^{2}$ Department of Gastroenterology, Sir Run Run Shaw Hospital, Hangzhou, Zhejiang 310016; \\ ${ }^{3}$ Department of Intensive Care Medicine, The Central Hospital of Jinhua City, Jinhua, Zhejiang 321001, P.R. China
}

Received June 12, 2014; Accepted September 15, 2014

DOI: $10.3892 /$ or.2014.3679

\begin{abstract}
The purpose of this study was to evaluate the anti-growth effect of the self-designed multiple antigenic polypeptide (MAP) vaccine comprising B-cell epitopes of heparanase (HPSE) on HCC97-H hepatocellular carcinoma (HCC) in mice. The polyclonal antibodies against the B-cell epitopes of HPSE were prepared by immunizing rabbits with freshly synthesized MAP vaccine. HCC-bearing models were constructed on BALB/c nude mice. Anti-MAP antibodies were administrered to the models to assess the effects on HPSE activity, HCC growth, the expression of VEGF/bFGF and the value of micro-vessel density (MVD). The anti-MAP antibodies were harvested, purified and identified. These antibodies were able to specifically bind with the dominant epitopes of the precursor protein and large subunit monomer of HPSE, decrease HPSE activity, suppress the expressions of VEGF and bFGF, reduce the MVD, and markedly shrink the HCC volume. Based on these findings, MAP vaccine based on the B-cell epitopes of HPSE seemed to provide theoretical evidence for further study of the synthesized HPSE MAP vaccine in the treatment of HCC.
\end{abstract}

\section{Introduction}

As a consequence of immune tolerance, primary liver cancer patients usually do not have a good response to tumor cells (1). Exogenous immunization aims to disrupt such intolerance and enhance the immune response (1). However, the therapeutic outcomes of most existing immunotherapeutic approaches

Correspondence to: Professor Jianmin Yang, Department of Gastroenterology, Zhejiang Provincial People's Hospital, 158 Shangtang Road, Hangzhou, Zhejiang 310014, P.R. China

E-mail: zjhealth@tom.com

${ }^{*}$ Contributed equally

Key words: heparanase, multiple antigenic peptide, epitope, hepatocellular carcinoma on hepatocellular carcinoma (HCC) usually are not satisfactory, mainly due to poor immunogenicity, the phenomenon of immune suppression and the interference of regulatory T cells (2-5).

As a novel approach of antitumor immunotherapy, epitope peptide vaccine has been developed rapidly in recent years. Compared with other vaccines, peptide vaccine has significant advantages such as simple composition, targeted immune response and exemption of pathological epitopes $(6,7)$. However, its smaller molecular weight and simple structure often lead to a decreased immunogenicity and an unsatisfactory immune response, especially the humoral immune response $(7,8)$. To resolve this problem, the multiple antigenic polypeptide (MAP) design has been suggested in recent years, which simulates the natural epitope structure well and eliminates the carrier protein (9). Theoretically, the MAP design can arouse humoral immunity in vivo, inducing highly specific polyclonal antibodies.

Besides the structural design, it is also essential to choose an ideal tumor associate antigen (TAA) as the therapeutic target for epitope peptide vaccines. As a promising TAA in immunotherapeutic areas, heparanase (HPSE) has been investigated in recent years (10). The HPSE precursor protein has a molecular weight of $\sim 65 \mathrm{kDa}$. It is a heterodimer consisting of two subunits, with a molecular weight of 50 and $8 \mathrm{kDa}$. The former weight represents its mature activated form (10). HPSE is currently the only endoglycosidase identified that can specifically degrade the heparan sulfate (HS) side chain of heparan sulfate proteoglycans (HSPG) in the ECM or at BM, resulting in the destruction of ECM or BM, release of multiple types of cytokines such as bFGF and VEGF and facilitation of malignant angiogenesis and tumor growth (11-13). HPSE is overexpressed in most tumors, including in $\mathrm{HCC}$, and plays a key role in cancer growth and metastasis (14). While HPSE is expressed at a relatively low level in mammalian lymphoid organs, leukocytes and platelets, it is hardly expressed in other normal tissues. Therefore, HPSE may be regarded as an important TAA as well as a target molecule in antitumor treatment $(15,16)$. Certain HPSE inhibitors such as antisense nucleic acid, siRNA and antibodies against its $50 \mathrm{kDa}$ large subunits may effectively suppress the growth and metastasis of malignant tumors (17-22). However, evident defects of these inhibitors, such as heterology, being easily degradable, having 
a short aging time and poor maneuverability in clinical treatment were also identified $(17,19)$.

Based on human HPSE protein structure and its predicted B-cell epitopes via bioinformatics, we designed and synthesized the MAP vaccine, and validated that it induced specific anti-MAP polyclonal antibodies in vivo $(23,24)$. In this study, to investigate the immunotherapeutic effect on human HCC growth, specific antibodies induced by the self-synthesized MAP were administered to tumor-bearing BALB/c nude mice through passive immunity. Our results suggest that the synthesized HPSE B-cell epitope-based MAP vaccine effectively limited HCC growth in vivo, by virtue of its anti-MAP polyclonal antibodies. Our study provides theoretical evidence for additional studies on the MAP vaccine composed of HPSE B-cell epitopes in the treatment of HCC.

\section{Materials and methods}

Experimental animals and cells. Four-week-old pathogen-free male BALB/c nude mice (weighing $20 \pm 2 \mathrm{~g}$, SPF grade, certificate no. SCXK20130198) were purchased from the Shanghai SLAC Laboratory Animal Co., Ltd. (Shanghai, China). White-hair-black-eye (WHBY) rabbits, derived from Japanese big-ear white rabbits, were provided by Animal Experimental Center of Zhejiang Chinese Medical University (Hangzhou, China). Animals were kept at the Animal Research Center of Zhejiang Chinese Medical University and provided with water and food ad libitum. The animal experiments were approved by the Ethics Committee of the Zhejiang Chinese Medical University. Invasive manipulations were performed under sodium pentobarbital anesthesia, and all efforts were made to minimize suffering. The HCC cell line HCC97-H (HPSE-positive) was purchased from the Liver Cancer Institute of Zhongshan Hospital (Shanghai, China), maintained at our laboratory under conditions of $37^{\circ} \mathrm{C}$ and $5 \% \mathrm{CO}_{2}$, and was routinely cultured in Dulbecco's modified Eagle's medium (DMEM) supplemented with penicillin (100 U/ml), streptomycin $(100 \mu \mathrm{g} / \mathrm{ml})$ and $10 \%$ fetal bovine serum (FBS).

Preparation of HPSE B-cell epitope-based MAP vaccine. The MAP vaccine composed of the B-cell epitope of HPSE was synthesized, purified, and identified as described previously $(23,24)$. Briefly, according to the amino acid sequence of human HPSE, peptide 'HCTNTDNPRYKEGDL' (279-293) of HPSE was selected as the dominant B-cell epitope by DNAStar software and BcePred online predication tool. The MAP vaccine was constructed by fusing 8 copies of epitope peptide 'HCTNTDNPRYKEGDL' to 5 copies of lysine $(\mathrm{K})$ by the Chinese Peptide Co. (Hangzhou, China). The schematic drawing of the self-designed MAP structure is provided in Fig. 1. The synthesized MAP was purified using reverse-phase high-performance liquid chromatography (HPLC) on a Vydac C18 column. The purity of the MAP was confirmed by analytic HPLC.

Harvest of specific anti-MAP antiserum. WHBY rabbits were immunized with the MAP vaccine, and its antiserum was isolated and identified according to the procedure we described previously (24). Briefly, eight-branched self-designed MAP was used to actively immunize WHBY rabbit intravenously

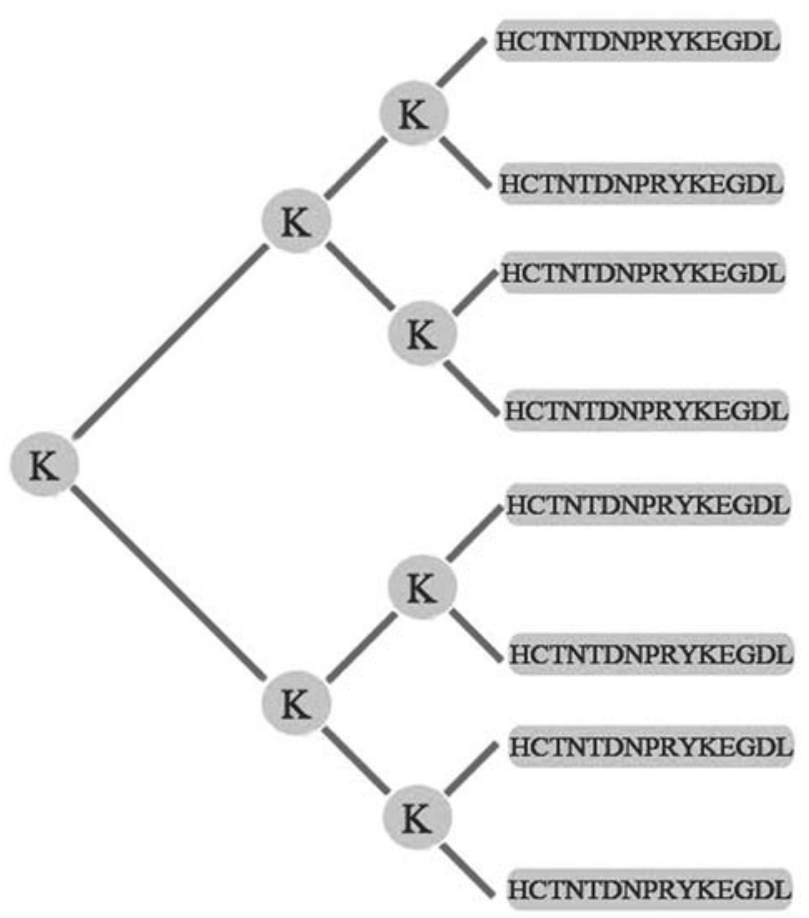

Figure 1. Schematic diagram of the self-synthesized multiple antigenic polypeptide (MAP) vaccine composed of B-cell epitopes of heparanase. Peptide 'HCTNTDNPRYKEGDL' (279-293) of heparanase was selected as the dominant B-cell epitope, and the MAP vaccine was constructed by fusing 8 copies of epitope peptide 'HCTNTDNPRYKEGDL' to 5 copies of lysine (K) by the Solid-Phase Peptide Synthesizer adopting eight-branched design.

four times, with an interval of 2 weeks. Freund's complete adjuvant was used in the prime immunization, while Freund's incomplete adjuvant (both from Sigma-Aldrich, St. Louis, MO, USA) and Th linear peptide (Chinese Peptide Co.) were used to boost the immunization. Serum samples were taken before the first immunization and 10 days after each manipulation, 5 times in total. For each sample, a standardized indirect ELISA assay was performed to mensurate the titers of polyclonal anti-MAP antibodies. After the entire immunizing procedure, WHBY rabbits were sacrificed and abundant antisera were harvested. The polyclonal antibodies against MAP vaccine contained in the antiserum were purified by caprylic acid/ammonium sulfate (CA-AS) precipitation, which is a classical, efficient and low-cost purification method for humoral antibodies (25). The concentration of the specific anti-MAP antibodies was determined by the Coomassie Brilliant Blue (Boster Biotechnology, China) assay as mentioned in our previous studies (24).

Binding affinity test. The ELISA assay was carried out to evaluate the affinity between the synthesized MAP and commercialized HPSE antibody (Takara Bio, Inc., Tokyo, Japan). Briefly, 96-well ELISA plates were coated with MAP at the concentration of $2 \mu \mathrm{g} / 100 \mu \mathrm{l}$ and $4 \mu \mathrm{g} / 100 \mu \mathrm{l}$, respectively and maintained overnight at $4^{\circ} \mathrm{C}$. After blocking using FBS, $0.05 \mu \mathrm{g} / 100 \mu \mathrm{l} /$ well of the commercialized HPSE antibodies were added, while unimmunized rabbit serum with a dilution of 1:5,000 was used as a negative control. The results were indicated by the mean OD value of triple wells. 
Western blot assay and protein electrophoresis. To identify the specificity of the anti-MAP antibodies, western blot analysis and protein electrophoresis were performed according to the manufacturer's instructions (KangChen Bio-Tech Inc., Shanghai, China). Specific immunoreactive bands became evident when the specific antibodies contained in the antiserum reacted with the HPSE of HCC97-H cells. Briefly, HCC97-H cells $\left(4 \times 10^{7}\right)$ were lysed and proteins were extracted offhandedly. An SDS-PAGE was produced. The commercialized rabbit anti-human HPSE antibody (InSight Biopharmaceuticals Ltd., Rehovot, Israel) with a dilution of 1:200, or the purified rabbit anti-MAP antiserum with a dilution of 1:3,000 served as the primary antibody. The unimmunized rabbit serum was used as a negative control. Immunoreactive bands were demonstrated using chemiluminescence.

HPSE activity suppression test. To evaluate the enzymatic activity of HPSE, the specific anti-MAP polyclonal antibodies contained in the rabbit antiserum were cultured together with HCC97-H cells in vitro. The HPSE activity was determined by detecting the amount of remaining HS substrate after digestion of the enzyme-substrate reaction. HPSE activity was calculated by measuring the absorbance at $450 \mathrm{~nm}$, which indicates the content of HS not being degraded by HPSE. The test was conducted as indicated in the user manual of the HPSE activity kit (Takara Bio, Inc.). Briefly, 1.0 U/well of HPSE Standard was added to a 96-well plate and incubated at a final concentration of $0 \mu \mathrm{g} / \mathrm{ml}$ (blank control), 50 and $100 \mu \mathrm{g} / \mathrm{ml}$ of anti-MAP antibodies, or $100 \mu \mathrm{g} / \mathrm{ml}$ of unimmunized rabbit $\mathrm{IgG}$ as a negative control in the nutrient medium for $1 \mathrm{~h}$ at $37^{\circ} \mathrm{C}$. Subsequently, HCC97-H cells (7x10 /well) were cultured in a 24-well plate for $48 \mathrm{~h}$, then treated with MAP-induced specific antibodies at the final concentration of $0 \mu \mathrm{g} / \mathrm{ml}$ (blank control), 50 and $100 \mu \mathrm{g} / \mathrm{ml}$, respectively, or $100 \mu \mathrm{g} / \mathrm{ml}$ of unimmunized rabbit IgG as a negative control in culture medium for $1 \mathrm{~h}$ at $37^{\circ} \mathrm{C}$. The supernatant of the nutrient medium was scraped out to measure the HPSE activity to evaluate the inhibitive effect of the anti-MAP antibodies on the HPSE enzymatic activity of HCC97-H cells.

Establishment of tumor-bearing murine model. The human HCC $97-H$ cells $\left(1 \times 10^{7} /\right.$ mouse) at logarithmic growth period were inoculated into the right flank of $10 \mathrm{BALB} / \mathrm{c}$ nude mice. Approximately six weeks later, the HCC97-H cells in five mice developed into solid tumors of $\sim 1 \mathrm{~cm}^{3}$, which were entirely gouged out subsequently and were immediately immersed into normal saline. Connective tissues were wrinkled and the lumps were sheared into $\sim 2 \mathrm{~mm}^{3}$ sections. The sections were subcutaneously implanted into the dorsal skin in $30 \mathrm{BALB} / \mathrm{c}$ nude mice, which were randomly divided into the small-dose immunizing (SDI) group, large-dose immunizing (LDI) group, and the control group, with 10 mice in each group. Four weeks later (before the beginning of passive immunization), B ultrasonography equipped with a water bag was performed to measure the implanted tumor sizes, ensuring there were no significant differences in size among the three groups. The mean tumor volume was measured and calculated according to the formula: $v=a b^{2} / 2$ (' $a$ ' is the maximum diameter of the tumor, and ' $b$ ' is the vertical diameter of the maximum diameter).
Passive immunization. After the HCC-bearing murine models were established (measured by B ultrasonography), MAP vaccine-derived polyclonal antibodies at the volume of $0.2 \mathrm{ml}$ were administered to the mice through the caudal vein on the SDI and LDI groups with the antibody dose of 5 and $10 \mathrm{mg} / \mathrm{kg}$, respectively, twice in total with an interval of two weeks. For the control group, $0.2 \mathrm{ml}$ unimmunized rabbit serum with a dilution of 1:100 was intravenously administered. B ultrasonography was implemented three weeks later to measure the HCC tumor volume, using the formula mentioned above. Six weeks after the passive immunization, HCC-bearing nude mice were sacrificed. The hypodermic xenograft was entirely gouged out, and its size was measured using a vernier caliper.

Immunohistochemical evaluation. At the end of the experiment, HCC-bearing nude mice were sacrificed. Histological sections of the hepatoma were manufactured and immunohistochemically evaluated for VEGF, bFGF and CD34 according to the manufacturer's instructions (Biotech Co., Ltd., Shanghai, China). Staining intensity of VEGF and bFGF was semiquantitatively evaluated according to a previously described method (24). Briefly, the determination of immunohistochemical results was blindly assessed by two senior pathologists. It was carried out firstly under low power lens (x100) to select the 'densely stained area', then 500 tumor cells were counted within five visual fields at a magnification of $\mathrm{x} 200$. The scoring standards of immunohistochemical staining were based on the coloring of the cancer cells and on the percentage of positive cells. Scoring for color was: no staining, 0 ; light yellow, 1; brown, 2; and dark brown, 3. Scoring for percentage was: negative, 0 ; the percentage of positive cells $\leq 10 \%, 1 ; 11-50 \%, 2$; and $\geq 51 \%, 3$. The intensity of VEGF or bFGF expression was denoted by the product of cancer cell staining intensity and positive cell percentage: $0-2,-; 3-4,+$; $5-7,++$; and $8-9,+++$.

CD34 immunostaining was used to represent mean vessel density (MVD), which was present in the endothelial cells of microvessels and could be stained as brown or brownish yellow. In this study, MVD was evaluated according to Weider's methodology (26): a high vascular density area was selected under low-power objective (x100), and the number of vascular stained by CD34 were counted in three visual fields under high-power microscope $(\mathrm{x} 400)$, and the average value was regarded as the MVD value of the tumor.

ELISA assay of serum VEGF and bFGF. Blood samples were taken from the murine orbits prior to euthanasia. Serum VEGF and bFGF concentrations were assessed by ELISA assay, according to the manufacturer's instructions (Cusabio, Wuhan, China). Briefly, $100 \mu \mathrm{l}$ standard, blank or sample was plated in triplicate per well in a 96-well plate and incubated for $2 \mathrm{~h}$ at $37^{\circ} \mathrm{C}$. Biotin-antibody working solution, horseradish peroxidase (HRP)-avidin working solution and 3,3',5,5'-tetramethylbenzidine (TMB) were subsequently added. Substrate was added to each well in turn and incubated in different environmental conditions. Stop solution was then added to each well when the first four wells containing the highest concentration of standards developed an obvious blue color. The optical density of each ELISA well was indicated by a microplate reader set to $450 \mathrm{~nm}$. Accordingly, the VEGF or 


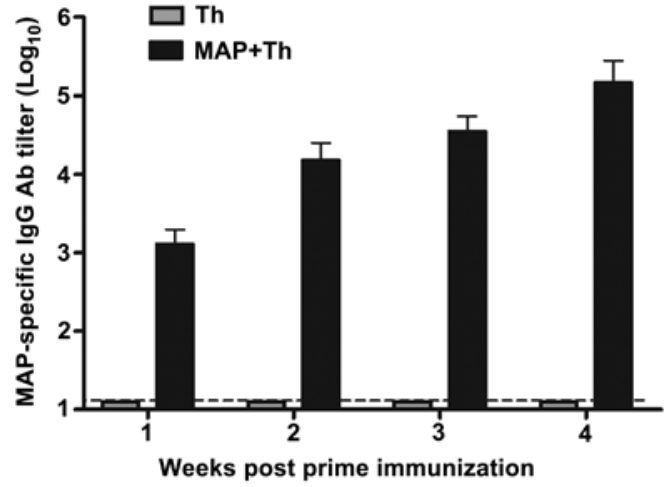

Figure 2. Multiple antigenic polypeptide (MAP)-specific antibody titers induced by heparanase B-cell epitope-based MAP vaccine. Rabbits were vaccinated using the self-synthesized MAP and Th linear peptide plus Freund's adjuvant. Sera were collected at 1,2,3 and 4 weeks post prime immunization, and MAP-specific IgG antibodies were detected in rabbit serum samples by standard indirect ELISA. Experiments were repeated three times, and the lower limit detection (1:20) is indicated by a dotted line.

bFGF value was calculated on the basis of the 'standard curve' drawn by the professional soft 'CurveExert 1.4'.

Statistical analysis. Experimental results were presented as mean \pm standard deviation (SD). Data were analyzed by the Student's t-test or one-way ANOVA analysis. Statistical significance was defined as $\mathrm{P}<0.05$. All statistical analyses were performed by SPSS 11.5 software (SPSS Inc., Chicago, IL, USA).

\section{Results}

HPSE B-cell epitope-based MAP immunization induced high titers of MAP-specific IgG antibodies. The synthesized MAP composed of HPSE B-cell epitopes was already identified in our previous studies, and the purity of the MAP polypeptides was $>95 \%$ determined by purification through $\operatorname{HPLC}(23,24)$. To evaluate humoral immune responses induced by MAP based on HPSE B-cell epitopes, WHBY rabbits were vaccinated with $200 \mu \mathrm{g}$ of self-synthesized MAP plus Freund's adjuvant and Th linear peptide as mentioned in Materials and methods, and MAP-specific IgG antibodies were detected in rabbit serum samples by standard indirect ELISA. The synthesized MAP induced strong MAP-specific IgG antibody responses, with the titer of $1: 10^{3} 1$ week following the first immunization, then the titer reached $\sim 1: 10^{4}$ prior to boost, and reached the highest peak over 1:10 2 weeks following the boost immunization (Fig. 2). By contrast, only the background level of antibody responses was detected in the rabbit serum immunized with Th linear peptide alone.

The synthesized MAP had high-binding affinity with commercialized HPSE antibody. Binding affinity between commercialized HPSE antibody and the synthesized MAP polypeptides was assessed by indirect ELISA assay. The coated HPSE B-cell epitope-based MAP of different concentrations reacted with the commercialized HPSE antibody (1:5,000 dilution) or the unimmunized rabbit serum (negative control, 1:5,000 dilution), respectively. The results showed that the synthesized MAP had high-binding affinity with the

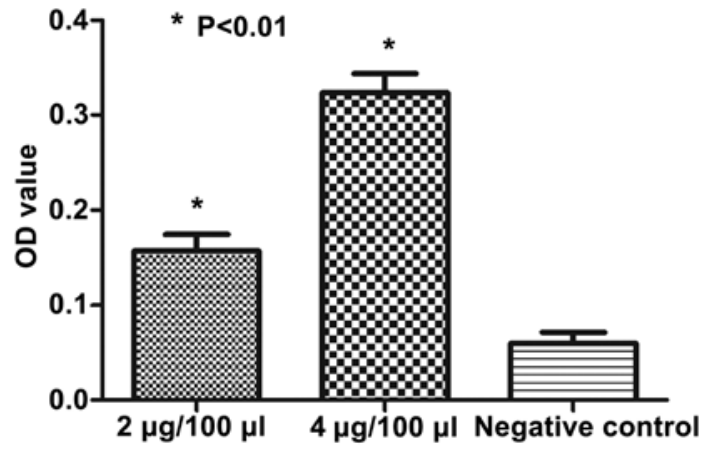

Figure 3. Binding affinity of the self-designed multiple antigenic polypeptide (MAP) composed of B-cell epitopes of heparanase. Ninety-six well ELISA plates were coated with MAP at the concentration of $2 \mu \mathrm{g} / 100 \mu \mathrm{l}$ and $4 \mu \mathrm{g} / 100 \mu \mathrm{l}$, respectively. After blocking with fetal bovine serum, $0.05 \mu \mathrm{g} / 100 \mu \mathrm{l} /$ well of the commercialized heparanase (HPSE) antibodies were added, while unimmunized rabbit serum with a dilution of 1:5,000 was used as a negative control. The results were indicated by the mean OD value of triple wells. The results showed that the synthesized MAP had high $(\mathrm{P}<0.01$, compared with the negative control) and dose-dependent $(\mathrm{P}<0.01$, compared between $2 \mu \mathrm{g} / 100 \mu \mathrm{l}$ and $4 \mu \mathrm{g} / 100 \mu \mathrm{l} \mathrm{MAP})$ binding affinity with the commercialized HPSE antibody.

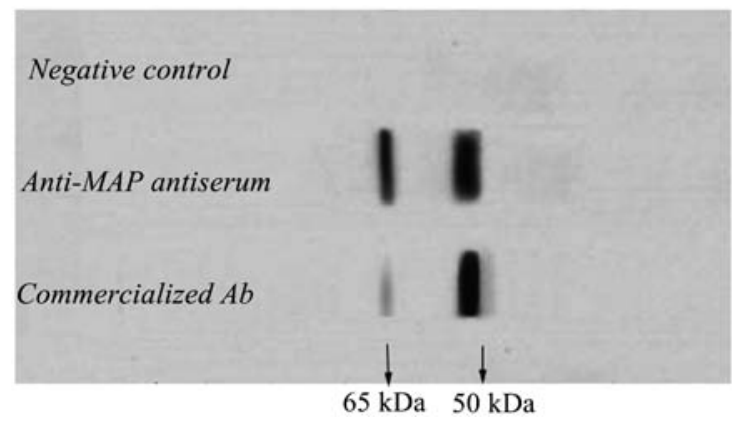

Figure 4. Electrophoresis and chemiluminescence outcomes of western blot assay. Total protein extracted from HCC97-H cells was reacted with the anti-multiple antigenic polypeptide (MAP) polyclonal antibodies. The primary antibody was the commercialized rabbit anti-human heparanase (HPSE) antibody with a dilution of 1:200, or the purified rabbit anti-MAP antiserum with a dilution of 1:3,000. The unimmunized rabbit serum was used as a negative control. Electrophoresis and chemiluminescence showed a clear band of $\sim 50 \mathrm{kDa}$ and a visible band of $65 \mathrm{kDa}$ in the commercialized antibody column. In the anti-MAP antiserum column, 65- and 50-kDa bands were markedly evident, while no band was observed in the corresponding locations in the negative control column.

commercialized HPSE antibody, compared with the negative control (Fig. 3).

Anti-MAP antibodies bound with 65- and 50-kDa HPSE protein. Protein extracted from HCC97-H cells was utilized to react with the anti-MAP polyclonal antibodies. The commercialized polyclonal rabbit anti-human HPSE antibody and the unimmunized rabbit serum were used as a positive and negative control, respectively. Electrophoresis and chemiluminescence showed a clear band of $\sim 50 \mathrm{kDa}$ and a visible band of $65 \mathrm{kDa}$ in the column of the positive control. In the anti-MAP antiserum column, 65- and 50-kDa bands were markedly exhibited (Fig 4). Nevertheless, no band was observed in both the corresponding locations in the negative control column. According to the instructions of the commercialized antibody, protein of the 65- and 50-kDa was most probably the precursor 

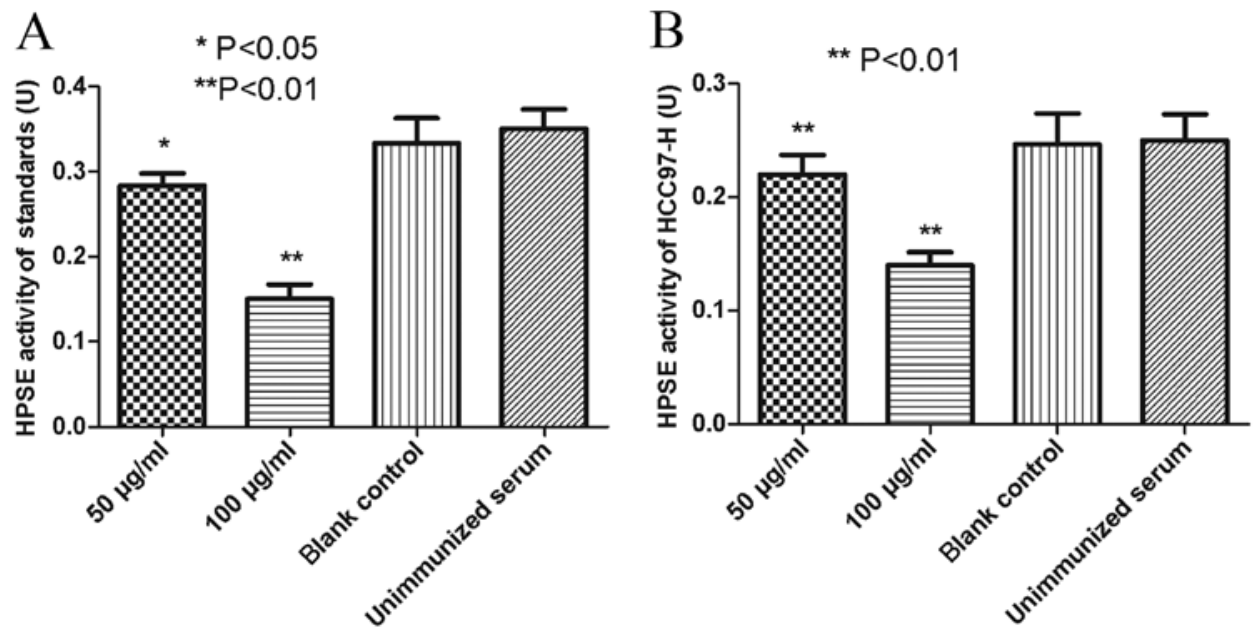

Figure 5. (A) Inhibitory effect of anti-multiple antigenic polypeptide (MAP) polyclonal antibodies contained in the immunized rabbit antiserum on heparanase enzymatic activity of the standards. After adding anti-MAP antibodies of 100 and $50 \mu \mathrm{g} / \mathrm{ml}$ to the cell culture medium containing heparanase (HPSE) standards and incubating for $1 \mathrm{~h}$ at $37^{\circ} \mathrm{C}$, the standards' of the HPSE activity decreased by 56.3\% ( $\left.\mathrm{P}<0.01\right)$ and $17.8 \%(\mathrm{P}<0.05)$, respectively, compared with the blank control group or the unimmunized rabbit serum control group. (B) Inhibitory impact of anti-MAP polyclonal antibodies contained in the immunized rabbit antiserum on HPSE enzymatic activity of the HCC97-H cells. After adding anti-MAP antibodies of 100 and $50 \mu \mathrm{g} / \mathrm{ml}$ to the nutrient medium containing $\mathrm{HCC} 97-\mathrm{H}$ cells and incubating for $1 \mathrm{~h}$ at $37^{\circ} \mathrm{C}$, HPSE cell activity decreased by $42.9 \%(\mathrm{P}<0.01)$ and $11.6 \%(\mathrm{P}<0.01)$, respectively, compared with the blank control group or the unimmunized rabbit serum control group.
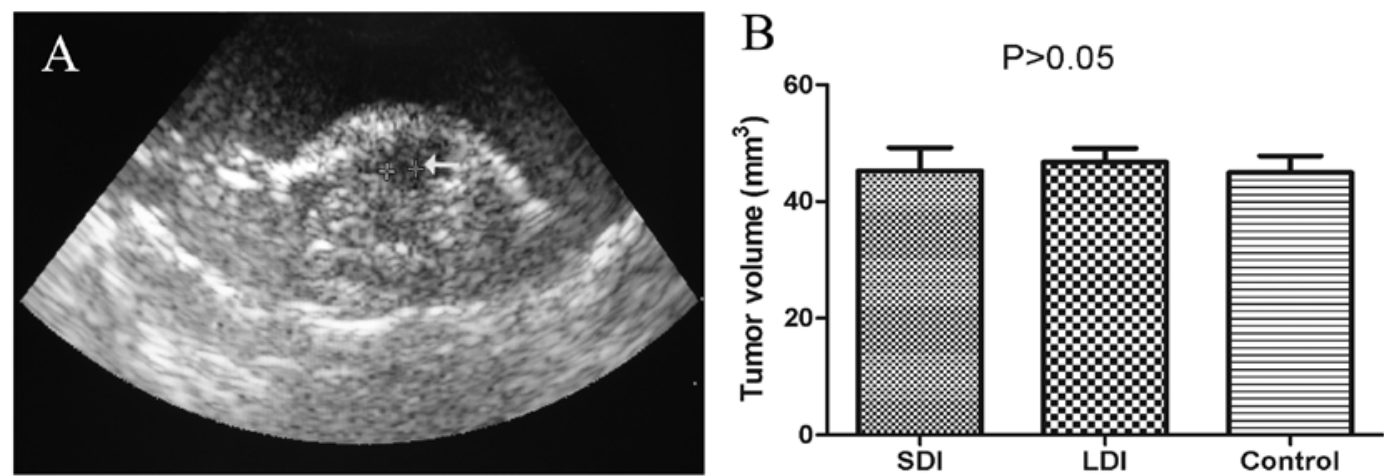

Figure 6. Xenograft volume four weeks posthepatocellular carcinoma (HCC) implanting. B ultrasonography was implemented four weeks after the HCC-implanting surgery. A representative ultrasonic image is shown in (A). (B) Ultrasonic measurement and the formula mentioned in Materials and methods, the mean tumor volumes of the small-dose immunizing (SDI), large-dose immunizing (LDI) and control groups were $45.3 \pm 6.8,46.7 \pm 4.2$ and $44.9 \pm 5.1 \mathrm{~mm}^{3}$, respectively. There were no statistical differences among the three groups $(\mathrm{P}>0.05)$.

of HPSE protein of HCC97-H cells and its large subunit, respectively.

Anti-MAP polyclonal antibodies elicited potent inhibitory effect on HPSE activity. When the HPSE standards were incubated with anti-MAP antibodies at a concentration of $100 \mu \mathrm{g} / \mathrm{ml}$, HPSE activity decreased by $56.3 \%$, compared with the blank control group or the negative control group $(\mathrm{P}<0.01)$. After the standards were treated with a final concentration of $50 \mu \mathrm{g} / \mathrm{ml}$ anti-MAP antibodies, a decrease of $17.8 \%$ on HPSE enzymatic activity was detected $(\mathrm{P}<0.05)$. No impact on the HPSE activity was observed under the treatment with a final anti-MAP antibody concentration of $0 \mu \mathrm{g} / \mathrm{ml}$ (blank control), compared with the negative control $(\mathrm{P}>0.05)$ (Fig. 5A).

To assess the impact on HPSE secreted by malignant HCC97-H cells, anti-MAP antibodies contained in the immunized rabbit antiserum were cultured with the HCC $97-\mathrm{H}$ cell line. After being incubated with anti-MAP antibodies at a final concentration of 100 and $50 \mu \mathrm{g} / \mathrm{ml}$, the HPSE activity of the culture supernatant decreased by 42.9 and $11.6 \%$, respectively, compared with the blank control or the negative control $(\mathrm{P}<0.01)$. The supernatant HPSE activity was not significantly altered following culture with the anti-MAP antibody of $0 \mu \mathrm{g} / \mathrm{ml}$ (blank control), compared with the negative control $(\mathrm{P}>0.05)$ (Fig. 5B).

Xenograft volume four weeks post-HCC implanting. The HCC-implanting operation was performed successfully and no mouse died during and after the surgery. B ultrasonography equipped with a water bag was implemented at the end of the 4th week after the surgical process. A representative ultrasonic image is provided in Fig. 6A. According to the outcomes of B ultrasonic measurement and the formula mentioned in Materials and methods, the mean tumor volumes of the SDI, LDI and control groups were $45.3 \pm 6.8,46.7 \pm 4.2$ and $44.9 \pm 5.1 \mathrm{~mm}^{3}$, respectively, and there was no statistical difference among the three groups $(\mathrm{P}>0.05)$ (Fig. 6B). 
A

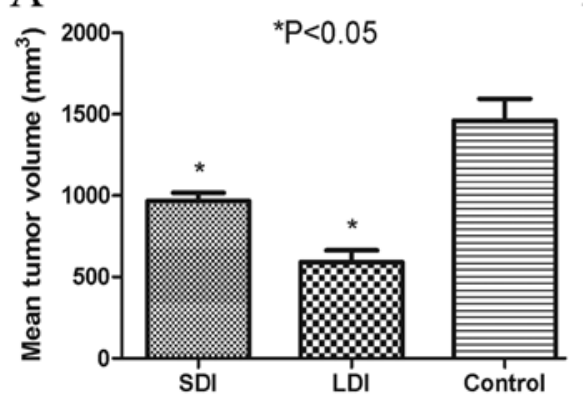

B

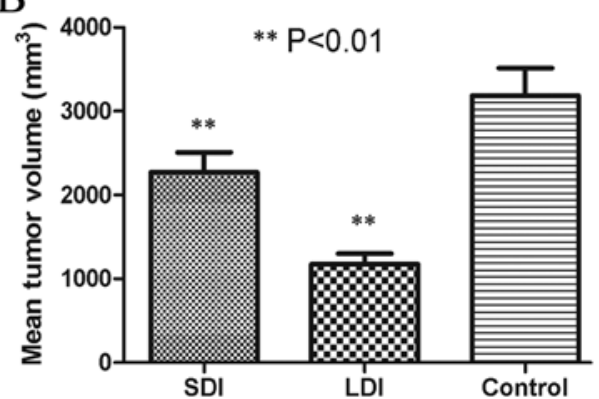

Figure 7. Hepatocellular carcinoma (HCC) volume three and six weeks after passive immunization. (A) Three weeks after passive immunization, B ultrasonography showed the mean tumor volume of the small-dose immunizing (SDI), large-dose immunizing (LDI) and control groups to be $967 \pm 58,592 \pm 96$ and $1,460 \pm 103 \mathrm{~mm}^{3}$, respectively, with significant differences between two groups ( $\left.\mathrm{P}<0.05\right)$. (B) Six weeks after passive immunization, the mean tumor volumes of the SDI, LDI and control groups were 2,270 $\pm 515,1,175 \pm 591$ and $3,188 \pm 366 \mathrm{~mm}^{3}$, respectively, as determined by vernier caliper. The mean tumor volume in the SDI or LDI group were significantly reduced, as compared with the control group $\left.{ }^{* *} \mathrm{P}<0.01\right)$.

HPSE B-cell epitope-based MAP-limited HCC growth in mice. After the tumor-bearing models were validated by B ultrasonography, MAP vaccine-derived polyclonal antibodies were injected to the mice in the SDI and LDI groups with different doses. For the control group, diluted unimmunized rabbit serum was administered. All the mice survived until the end of the experiment. Three weeks after passive immunization, B ultrasonography showed the mean tumor volume of the SDI, LDI and control groups to be $967 \pm 88$, $592 \pm 126$ and $1,460 \pm 233 \mathrm{~mm}^{3}$, respectively. Significant differences between two groups were observed $(\mathrm{P}<0.05)$ (Fig. 7A). Six weeks after passive immunization, the mean tumor volume of the SDI, LDI and control groups were 2,270 \pm 415 , $1,175 \pm 291$ and $3,188 \pm 566 \mathrm{~mm}^{3}$, respectively, as determined by vernier caliper. The mean tumor volume in the SDI or LDI group was significantly reduced, as compared with the control group $(\mathrm{P}<0.01)$ (Fig. 7B).

Immunohistochemical evaluation of VEGF, bFGF and $C D 34$. All tumor-bearing mice survived until the end of the experiment (six weeks after the first passive immunization). Histological HCC sections were manufactured, and immunohistochemical stainings of VEGF, bFGF and CD34 were performed. According to the scoring standard mentioned above, the expression of VEGF or bFGF in the SDI, LDI and control groups was,+++ and +++ , respectively (Table I). The mean value of MVD (represented by CD34 immunostaining) in the SDI, LDI and control groups was $21.64 \pm 5.79 /$ field, $13.48 \pm 4.31 /$ field and 29.67 $\pm 5.83 /$ field, respectively. There were significant differences among the groups $(\mathrm{P}<0.05)$ (Table I). Representative immunohistochemical images are shown in Fig. 8.

The anti-MAP antibody reduced serum concentrations of VEGF and bFGF. Serum VEGF and bFGF levels were evaluated by ELISA, which assisted the in vivo malignant cascades of HCC and were released mostly by HPSE enzymolysis $(10,12)$. The mean VEGF concentrations in the SDI, LDI and control groups were $113.85 \pm 19.48,58.81 \pm 20.26$ and $161.90 \pm 27.41 \mathrm{pg} / \mathrm{ml}$, respectively (Fig. 9A), while the bFGF levels in the corresponding groups were $91.80 \pm 23.59$, $35.47 \pm 13.32$ and $172.83 \pm 47.32 \mathrm{pg} / \mathrm{ml}$, respectively (Fig. 9B). The mean concentration of VEGF or bFGF in the SDI or LDI
Table I. Immunohistochemical evaluation of VEGF, bFGF and CD34.

\begin{tabular}{lccc}
\hline Groups & VEGF & bFGF & MVD \\
\hline SDI & ++ & ++ & $21.64 \pm 5.79 /$ field \\
LDI & + & + & $13.48 \pm 4.31 /$ field \\
Control & +++ & +++ & $29.67 \pm 5.83 /$ field
\end{tabular}

VEGF and bFGF were lowly expressed (+) in the LDI group $(10 \mathrm{mg} / \mathrm{kg}$ anti-MAP antibody), moderately expressed $(++)$ in the SDI group $(5 \mathrm{mg} / \mathrm{kg}$ anti-MAP antibody) and highly expressed (+++) in the control group (unimmunized rabbit serum). MVD count in the LDI or SDI group was significantly less than that in the control group $(\mathrm{P}<0.05)$. There was a significant difference between the SDI and LDI groups $(\mathrm{P}<0.05)$. LDI, large-dose immunizing; SDI, small-dose immunizing; MVD, micro-vessel density.

group was much lower than that in the control group $(\mathrm{P}<0.01)$. The serum level of VEGF or bFGF in the LDI group was also markedly lower than that in the SDI group $(\mathrm{P}<0.01)$, which showed a certain dose-dependent effect.

\section{Discussion}

Although there have been advances in preventive and therapeutic approaches, HCC remains one of the major causes of mortality worldwide. Over-growth, invasion and metastasis remain the major bewilderments in curing HCC patients, thus effective therapy to combat hepatoma is yet to be established $(27,28)$. To identify a complementary approach in the HCC therapeutic area of study, immunotherapy has been under investigation during the past few years. It utilizes the immune system to recognize and cope with tumor cells and has shown encouraging results in certain human clinical trials (4). The fundamental purpose of such therapy is to manufacture vaccines that elicit potent anti-growth or anti-metastatic immune responses without side effects.

The central issue in the development of HCC immunotherapeutic strategies is the identification of relevant TAA capable of mediating antitumor effects via a competent immune system. Investigators have advocated that the ideal TAA should be: 


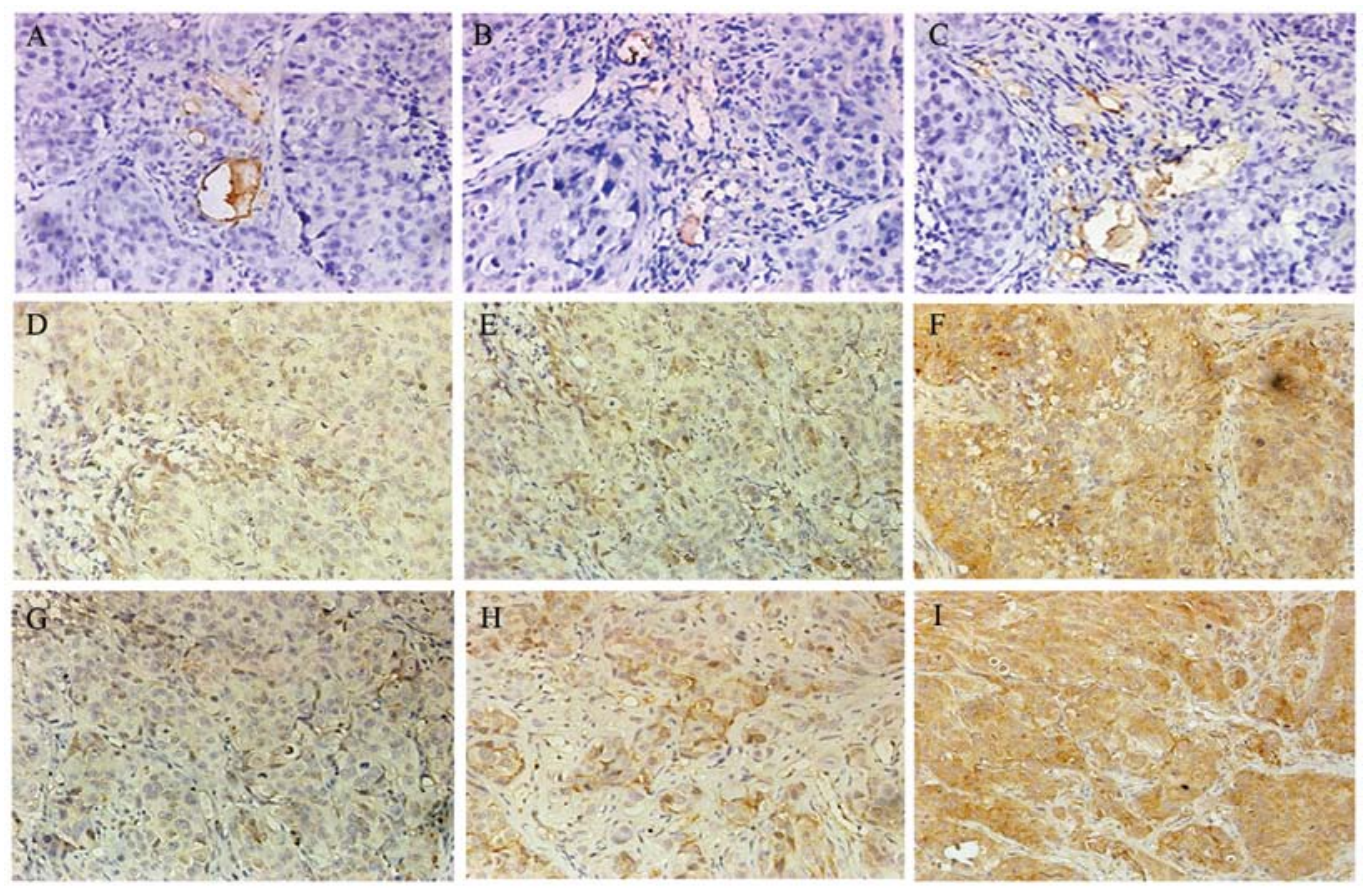

Figure 8. Immunohistochemical images of micro-vessel density (MVD), VEGF and bFGF. At the end of the experiment (six weeks after the first passive immunization), histological sections of hepatocellular carcinoma (HCC) were manufactured, and immunohistochemistry of VEGF, bFGF and CD34 was performed. (A-C) Representative MVD immunohistochemical stainings of the large-dose immunizing (LDI), small-dose immunizing (SDI) and control groups, respectively (x400). (D-F) Representative VEGF immunostainings of the LDI, SDI and control groups, respectively (x400). (G-I) Representative bFGF immunohistochemical images of the LDI, SDI and control groups, respectively (x400).
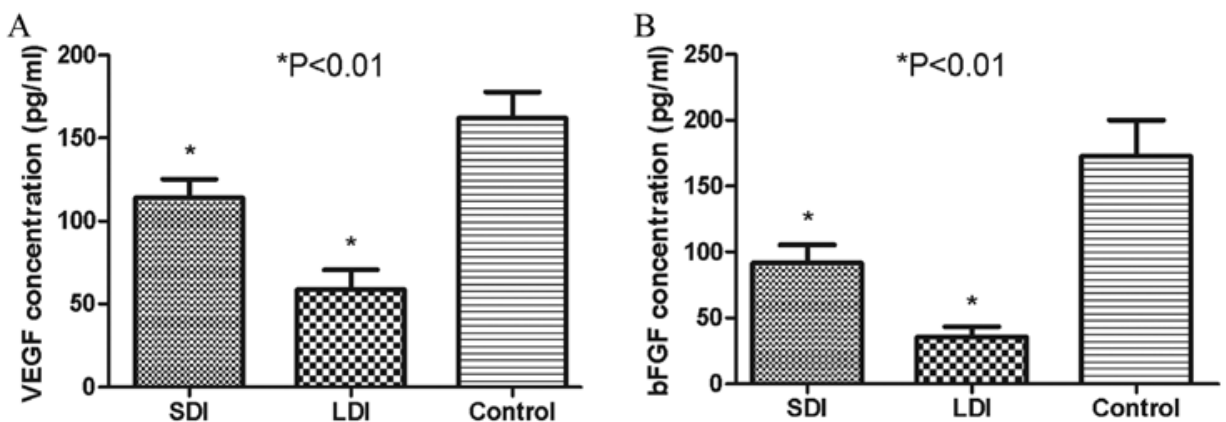

Figure 9. Antibodies induced by the multiple antigenic polypeptide (MAP) vaccine reduce serum concentrations of VEGF and bFGF. Serum VEGF and bFGF levels of tumor-bearing murine models were evaluated by ELISA. The mean VEGF concentrations in small-dose immunizing (SDI), large-dose immunizing (LDI) and control groups were $113.85 \pm 19.48,58.81 \pm 20.26$ and $161.90 \pm 27.41 \mathrm{pg} / \mathrm{ml}$, respectively (Fig. 9A), while the bFGF levels in the corresponding groups were $91.80 \pm 23.59,35.47 \pm 13.32$ and $172.83 \pm 47.32 \mathrm{pg} / \mathrm{ml}$, respectively (Fig. 9B). The mean concentration of VEGF or bFGF in the SDI or LDI group was much lower than that in the control group ("P<0.01). The serum level of VEGF or bFGF in the LDI group was also markedly lower than that in the SDI group ("P<0.01).

i) unique, distinctly different than in normal cells, ii) constitutively expressed during the cell cycle and, iii) the constitutive expression is essential for cell survival (29). Currently, a number of TAA have been identified and described (30). The appearance of antigen-loss or antigen-alteration mutations in tumor cells in response to immune pressure has also been highlighted and well described $(31,32)$. To circumvent this issue, a class of TAA termed Universal Tumor Antigens (UTAs) has been suggested that is hypothesized to induce antitumor immunity against a wide range of tumor types, and to have critical functional roles in tumor growth and development (33). Previous studies have been performed to determine promising UTAs prior to the identification of HPSE (34).

Unlike other TAA, HPSE is found to be highly expressed in most mammalian malignant tumors, and its expression has been associated with tumor growth, metastasis and angiogenesis (11). Some tumor cells can downregulate or mutate the TAA expression to evade immune surveillance (35). However, due to the crucial role of HPSE in tumor progression, the downregulation or mutation of its expression as a means of immune escape may itself have deleterious effects on the proliferation and metastasis of tumor cells. Tregs usually accumulate at the tumor site, where they suppress the function of effective lymphocytes and inhibit tumor growth (36). Notably, Tregs against HPSE were not found in patients with certain malignancies (37). HPSE-targeted immunotherapy is thus expected to be prolonged and more efficient owing to the absence of suppressive cells. Additionally, HPSE has been the sole endoglycosidase capable of vitiating the ECM and BM, by splitting the HS chain of glycosaminoglycan (10-16). As a consequence, 
activation of HPSE plays a key role in growth and invasion that enables tumor cells to break through the ECM and BE barriers, releasing multiple types of cytokine and causing the formation of new malignant vessels and the over-growth of tumors $(10-16,38)$. Some studies have demonstrated that certain HPSE inhibitors, such as polysaccharides, siRNA, and polypeptide antibodies, had the potency of suppressing tumor growth, invasion, metastasis and angiogenesis (13,17-24). Based on the above results, HPSE may be regarded as a promising and crucial target for antitumor immunological studies.

The first group of immunogenic nonapeptide epitopes of the HPSE amino acid sequence was previously identified by Sommerfeldt et al (16) approximately 10 years ago (26). In previous studies, we screened and identified that peptide fragment 279-293 was a dominant B-cell epitope of HPSE, and accordingly synthesized the MAP vaccine adopting the 8 -branched design $(23,24)$. In this study, we evaluated its immunological effects on hindering HCC growth in tumor-bearing nude mice.

The establishment of tumor-bearing murine models was a crucial process in this study. B ultrasonography was therefore carried out to ensure there were no significant differences in xenograft size among the three groups prior to administration, and the ultrasonic outcomes presented a fine uniformity in the tumor load in the SDI, LDI and control groups. To assess the anti-growth potency of the self-synthesized MAP composed of B-cell epitopes of HPSE, we administrered the tumor-bearing nude mice with the purified antiserum containing the anti-MAP polyclonal antibodies. As early as in 1981, this passive immunizing method applied on immunodeficient genetically engineered mice was approved as practical and safe by Katz et al (39), as long as the volume of heterogenic antiserum used in the immunization was $\leq 0.2 \mathrm{ml}$.

To determine the binding domain of HPSE protein reacting with the anti-MAP polyclonal antibodies contained in the immunized rabbit serum, western blot analysis with electrophoresis was conducted after the antiserum was isolated and purified. As a result, two clear bands located at $\sim 65$ and $50 \mathrm{kDa}$ were presented by chemiluminescence in the anti-MAP antiserum column, while no visible band was observed in the corresponding locations in the negative control column. The above findings indicate the specific antibodies induced by the synthesized MAP were probably bound with the dominant epitopes of the HPSE precursor protein and its large subunit monomer. It has been previously reported that HPSE polypeptide antibodies derived from the epitopes contained in the region of N-terminus of the 50-kDa large subunit may effectively block HPSE, reduce the amount of HPSE or slash the enzymatic activity (40). However, in this study and former studies $(23,24)$, the HPSE activity of HCC97-H cells was evidently also decreased when reacted with anti-MAP polyclonal antibodies induced by the self-designed MAP composed of B-cell eipitopes (279-293 of the large subunit) of HPSE, which was not present in the $\mathrm{N}$-terminus of the $50 \mathrm{kDa}$ large subunit.

To verify the anti-growth potency of the synthesized MAP, the specific anti-MAP antibodies induced by the HPSE B-cell epitope-based vaccine were intravenously immunized to the murine models bearing HCC xenografts, while the unimmunized rabbit serum was administrered to the control group. Three or six weeks after passive immunization, size-monitoring results demonstrated that $\mathrm{HCC}$ volumes in the SDI and LDI groups were significantly smaller than that in the control group. There were also significant differences between the SDI and LDI groups, which suggested that the specific antibodies induced by the self-synthesized MAP vaccine could markedly and dose-dependently limit the over-growth of HCC in mice.

The enzymolysis of HPSE could split HS in HSPG, release and activate HS-linking cytokines such as bFGF and VEGF, which are fundamental positive regulators of angiogenesis, stimulating the proliferation of endothelial cells and enhancing vascular permeability (41). Findings of previous studies have reported that VEGF and bFGF were closely correlated with HCC growth, angiogenesis and probably prognosis $(42,43)$. In this experiment, the content of VEGF and bFGF was semi-quantitatively assessed by calculating the percentage of positively immunostained tumor cells, and was quantitatively analyzed by ELISA. Immunohistochemistry demonstrated that the expression of VEGF or bFGF in the SDI and LDI groups was much lower than that in the control group, and VEGF and bFGF expressed significantly higher in the LDI than in the SDI group. ELISA results showed that the serous concentration of VEGF and bFGF in the LDI and SDI groups were significantly lower than that in the control group. The VEGF and bFGF levels in the LDI group were also significantly lower than that in the SDI group. To assess the impact of synthesized HPSE B-cell epitope-based MAP on the angiogenesis of HCC, we semi-quantitatively counted MVD in the immunochemical sections, which was deemed as the gold standard of angiogenesis and may be presented by CD34 immunostaining (44). The result showed that the MVD value in the LDI or SDI group was much smaller than that in the control group, and there was also a significant difference between the SDI and LDI groups. Based on the above findings, we hypothesize that the specific polyclonal antibodies induced by self-synthesized MAP composed of HPSE B-cell epitopes were able to inhibit the release and expression of VEGF and bFGF, reduce MVD, and therefore suppress the malignant angiogenesis and over-growth. Moreover, similar to the findings of $\mathrm{Xu}$ et al (45), we identified a certain positive correlation between VEGF/bFGF levels and MVD. In this correlation, the stronger the expression of VEGF or bFGF, the higher the MVD.

In conclusion, our experiment suggests that the self-designed MAP vaccine composed of B-cell epitopes of human HPSE is capable of limiting human HCC growth in mice, which is probably induced by suppressing HPSE activity and tumor-associated angiogenesis, by virtue of its specific anti-MAP polyclonal antibodies. This study provides theoretical evidence for further study of the synthesized HPSE MAP vaccine in treating HCC.

\section{Acknowledgements}

This study was supported by the Zhejiang Medicine and Public Health Research Program (no. 2013KYA014) and National Natural Science Foundation of China (81400682). The authors would like to thank Professor HouQuan Tao for his excellent technical assistance. 


\section{References}

1. Llovet JM and Bruix J: Molecular targeted therapies in hepatocellular carcinoma. Hepatology 48: 1312-1327, 2008.

2. Rabinovich GA, Gabrilovich D and Sotomayor EM: Immunosuppressive strategies that are mediated by tumor cells Annu Rev Immunol 25: 267-296, 2007.

3. Ostrand-Rosenberg S: Immune surveillance: a balance between protumor and antitumor immunity. Curr Opin Genet Dev 18 $11-18,2008$.

4. Borghaei H, Smith MR and Campbell KS: Immunotherapy of cancer. Eur J Pharmacol 625: 41-54, 2009.

5. Zarour HM and Ferrone S: Cancer immunotherapy: Progress and challenges in the clinical setting. Eur J Immunol 41: 1510-1515, 2011.

6. Van der Burg SH, Bijker MS, Welters MJ, et al: Improved peptide vaccine strategies, creating synthetic artificial infections to maximize immune efficacy. Adv Drug Deliv Rev 58: 916-930, 2006.

7. Bijker MS, Melief CJ, Offringa R and van der Burg SH: Design and development of synthetic peptide vaccines: past, present and future. Expert Rev Vaccines 6: 591-603, 2007.

8. Brunsvig PF, Kyte JA, Kersten C, et al: Telomerase peptide vaccination in NSCLC: a phase II trial in stage III patients vaccinated after chemoradiotherapy and an 8-year update on a phase I/II trial. Clin Cancer Res 17: 6847-6857, 2011.

9. Joshi VG, Dighe VD, Thakuria D, et al: Multiple antigenic peptide (MAP): a synthetic peptide dendrimer for diagnostic, antiviral and vaccine strategies for emerging and re-emerging viral diseases. Indian J Virol 24: 312-320, 2013.

10. Vlodavsky I, Ilan N, Naggi A and Casu B: Heparanase: structure, biological functions, and inhibition by heparin-derived mimetics of heparin sulfate. Curr Pharm Des 13: 2057-2073, 2007.

11. Vlodavsky I, Elkin M, Abboud-Jarrous G, et al: Heparanase: one molecule with multiple functions in cancer progression. Connect Tissue Res 49: 207-210, 2008.

12. Roy M and Marchetti D: Cell surface heparan sulfate released by heparanase promotes melanoma cell migration and angiogenesis J Cell Biochem 106: 200-209, 2009.

13. Nasser NJ, Avivi A, Shafat I, et al: Alternatively spliced Spalax heparanase inhibits extracellular matrix degradation, tumor growth, and metastasis. Proc Natl Acad Sci USA 106: 2253-2258, 2009.

14. Vlodavsky I, Goldshmidt O, Zcharia E, et al: Mammalian heparanase: involvement in cancer metastasis, angiogenesis and normal development. Semin Cancer Biol 12: 121-129, 2002.

15. McKenzie EA: Heparanase: a target for drug discovery in cancer and inflammation. Br J Pharmacol 151: 1-14, 2007.

16. Sommerfeldt N, Beckhove $\mathrm{P}, \mathrm{Ge} \mathrm{Y}$, et al: Heparanase: a new metastasis-associated antigen recognized in breast cancer patients by spontaneously induced memory $\mathrm{T}$ lymphocytes. Cancer Res 66: 7716-7723, 2006.

17. Miao HQ, Liu H, Navarro E, et al: Development of heparanase inhibitors for anti-cancer therapy. Curr Med Chem 13: 2101-2111, 2006.

18. Tang XD, Wan Y, Chen L, et al: H-2Kb-restricted CTL epitopes from mouse heparanase elicit an antitumor immune response in vivo. Cancer Res 68: 1529-1537, 2008.

19. Liang XJ, Yuan L, Hu J, et al: Phosphomannopentaose sulfate (PI-88) suppresses angiogenesis by downregulating heparanase and vascular endothelial growth factor in an oxygen-induced retinal neovascularization animal model. Mol Vis 18: 1649-1657, 2012.

20. Dredge K, Hammond E, Handley P, et al: PG545, a dual heparanase and angiogenesis inhibitor, induces potent anti-tumour and anti-metastatic efficacy in preclinical models. Br J Cancer 104: 635-642, 2011

21. He X, Brenchley PE, Jayson GC, et al: Hypoxia increases heparanase-dependent tumor cell invasion, which can be inhibited by antiheparanase antibodies. Cancer Res 64: 3928-3933, 2004.

22. Borsig L, Vlodavsky I, Ishai-Michaeli R, et al: Sulfated hexasaccharides attenuate metastasis by inhibition of P-selectin and heparanase. Neoplasia 13: 445-452, 2011.

23. Du L, Wang HJ, Yang JM, et al: T-helper epitope peptide improves immunological effects of the B cell epitopes of human heparanase protein. Chin J Microbiol Immunol 28: 869-872, 2008.
24. Yang JM, Wang HJ, Du L, et al: Screening and identification of novel B cell epitopes in human heparanase and their anti-invasion property for hepatocellular carcinoma. Cancer Immunol Immunother 58: 1387-1396, 2009.

25. Liu X, Cai M, Wang X and Li X: One simple and efficient method for purification of IgG McAb from mice ascites: caprylic acid/ ammonium sulfate precipitation. Hua Xi Yi Ke Da Xue Xue Bao 30: 455-456, 1999 (In Chinese).

26. Weidner $\mathrm{N}$ : Intratumor microvessel density as a prognostic factor in cancer. Am J Pathol 147: 9-19, 1995.

27. Bridges JF, Dong L, Gallego G, et al: Prioritizing strategies for comprehensive liver cancer control in Asia: a conjoint analysis. BMC Health Serv Res 12: 376, 2012.

28. Aravalli RN, Cressman EN, Steer CJ, et al: Cellular and molecular mechanisms of hepatocellular carcinoma: an update. Arch Toxicol 87: 227-247, 2013.

29. Darzynkiewicz Z: Will cancer immunotherapy fail? Scientist 20: 14, 2006.

30. Kratky W, Reis e Sousa C, Oxenius A and Spörri R: Direct activation of antigen-presenting cells is required for $\mathrm{CD}^{+} \mathrm{T}$-cell priming and tumor vaccination. Proc Natl Acad Sci USA 108 17414-17419, 2011.

31. Koop A, Sellami N, Adam-Klages S, et al: Down-regulation of the cancer/testis antigen 45 (CT45) is associated with altered tumor cell morphology, adhesion and migration. Cell Commun Signal 11: 41, 2013.

32. Weldon JE, Xiang L, Zhang J, et al: A recombinant immunotoxin against the tumor-associated antigen mesothelin reengineered for high activity, low off-target toxicity, and reduced antigenicity. Mol Cancer Ther 12: 48-57, 2013.

33. Vonderheide RH: Universal tumor antigens for cancer vaccination: targeting telomerase for immunoprevention. Discov Med 7: 103-108, 2007.

34. Zhang YF, Tang XD, Gao JH, et al: Heparanase: a universal immunotherapeutic target in human cancers. Drug Discov Today 16: 412-417, 2011.

35. Palena $\mathrm{C}$ and Schlom J: Vaccines against human carcinomas: strategies to improve antitumor immune responses. J Biomed Biotechnol 2010: 380697, 2010.

36. Zou W: Regulatory T cells, tumour immunity and immunotherapy. Nat Rev Immunol 6: 295-307, 2006.

37. Bonertz A, Weitz J, Pietsch DH, et al: Antigen-specific Tregs control $\mathrm{T}$ cell responses against a limited repertoire of tumor antigens in patients with colorectal carcinoma. J Clin Invest 119: 3311-3321, 2009

38. Ilan N, Elkin M, Vlodavsky I, et al: Regulation, function and clinical significance of heparanase in cancer metastasis and angiogenesis. Int J Biochem Cell Biol 38: 2018-2039, 2006.

39. Katz M, Lynn M, Solotorovsky M, et al: Serological and biological activities of anti-Haemophilus influenzae ribosomal serum. Infect Immun 31: 1125-1131, 1981.

40. Levy-Adam F, Abboud-Jarrous G, Guerrini M, et al: Identification and characterization of heparin/heparan sulfate binding domains of the endoglycosidase heparanase. J Biol Chem 280: 20457-20466, 2005.

41. Fidler IJ and Ellis LM: The implications of angiogenesis for the biology and therapy of cancer metastasis. Cell 79: 185-188, 1994.

42. An FQ, Matsuda M, Fujii H and Matsumoto Y: Expression of vascular endothelial growth factor in surgical specimens of hepatocellular carcinoma. J Cancer Res Clin Oncol 126: 153-160, 2000.

43. Poon RT, Ng IO, Lau C, et al: Serum vascular endothelial growth factor predicts venous invasion in hepatocellular carcinoma: a prospective study. Ann Surg 233: 227-235, 2011.

44. Yang P, Yuan W, He J, et al: Overexpression of EphA2, MMP-9, and MVD-CD34 in hepatocellular carcinoma: implications for tumor progression and prognosis. Hepatol Res 39: 1169-1177, 2009.

45. Xu YZ, Zhu Y, Shen ZJ, et al: Significance of heparanase-1 and vascular endothelial growth factor in adrenocortical carcinoma angiogenesis: potential for therapy. Endocrine 40: 445-451, 2011. 